The design and construction of a prototype lateral-transfer retro-reflector for inter-satellite laser ranging

This content has been downloaded from IOPscience. Please scroll down to see the full text. 2014 Class. Quantum Grav. 31095015

(http://iopscience.iop.org/0264-9381/31/9/095015)

View the table of contents for this issue, or go to the journal homepage for more

Download details:

IP Address: 194.94.224.254

This content was downloaded on 11/06/2014 at 06:46

Please note that terms and conditions apply. 


\title{
The design and construction of a prototype lateral-transfer retro-reflector for inter-satellite laser ranging
}

\author{
R L Ward ${ }^{1}$, R Fleddermann ${ }^{1}$, S Francis ${ }^{1}$, C Mow-Lowry ${ }^{1,2}$, \\ D Wuchenich ${ }^{1}$, M Elliot $^{1}$, F Gilles $^{3}$, M Herding ${ }^{3}$, K Nicklaus $^{3}$, \\ J Brown $^{4}$, J Burke ${ }^{5}$, S Dligatch ${ }^{4}$, D Farrant ${ }^{4}$, K Green $^{4}$, \\ J Seckold $^{4}$, M Blundell ${ }^{6}$, R Brister ${ }^{6}$, C Smith ${ }^{6}$, \\ K Danzmann $^{2}$, G Heinzel ${ }^{2}$, D Schütze ${ }^{2}$, B S Sheard ${ }^{2}$, \\ W Klipstein ${ }^{7}$, D E McClelland ${ }^{1}$ and D A Shaddock ${ }^{1}$
}

${ }^{1}$ The Australian National University, Canberra ACT 0200, Australia

${ }^{2}$ Max Planck Institute for Gravitational Physics, (Albert Einstein Institute) and Institute for Gravitational Physics, Leibniz Universität Hannover, Callinstr 38, D-30167 Hannover, Germany

${ }^{3}$ SpaceTech GmbH, Seelbachstr. 13, D-88090 Immenstaad, Germany

${ }^{4}$ CSIRO Materials Science and Engineering, Lindfield NSW 2070, Australia

${ }^{5}$ BIAS, Optical Metrology and Opto-electronic Systems, Klagenfurter Str 2,

D-28359 Bremen, Germany

${ }^{6}$ EOS Space Systems, Mt. Stromlo Observatory, Weston Creek ACT 2611, Australia

${ }^{7}$ Jet Propulsion Laboratory, Pasadena, California, CA 91109, USA

E-mail: robert.ward@anu.edu.au

Received 13 February 2014, revised 14 March 2014

Accepted for publication 18 March 2014

Published 16 April 2014

\begin{abstract}
The Gravity Recovery and Climate Experiment (GRACE) mission, launched in 2002, is nearing an end, and a continuation mission (GRACE Followon) is on a fast-tracked development. GRACE Follow-on will include a laser ranging interferometer technology demonstrator, which will perform the first laser interferometric ranging measurement between separate spacecraft. This necessitates the development of lightweight precision optics that can operate in this demanding environment. In particular, this beam routing system, called the triple mirror assembly, for the GRACE Follow-on mission presents a significant manufacturing challenge. Here we report on the design and construction of a prototype triple mirror assembly for the GRACE Follow-on mission. Our constructed prototype has a co-alignment error between the incoming and
\end{abstract}


outgoing beams of $9 \mu \mathrm{rad}$, which meets the requirement that this error must be less than $10 \mu \mathrm{rad}$.

Keywords: satellite interferometry, gravity measurement, precision optics, satellite optics

PACS numbers: 7.87.+v, 07.60.Ly, 42.15.Eq, 42.79.Fm, 93.85.Hj

(Some figures may appear in colour only in the online journal)

\section{Introduction}

The Gravity Recovery and Climate Experiment (GRACE) mission [1, 2], launched in 2002, has provided over a decade of monthly measurements of the Earth's geoid. The time variation in the geoid has yielded significant insight into the movement and distribution of water over the entire surface of the Earth. The GRACE mission has had such a large scientific payoff [3-5] that a follow-on mission [6] has been approved to continue the data collection that will otherwise stop when the GRACE satellites inevitably fail (they were planned with a five-year lifespan).

The GRACE mission consists of two identical satellites flying in a near polar orbit, with one satellite following the other at a distance of $200 \mathrm{~km}$. The distance between the satellites is continuously measured with a $K$-band microwave ranging instrument providing micrometre level sensitivity, and the geoid is inferred from this time-series measurement. The GRACE Follow-on mission is due to launch in 2017. In order to minimize gaps in the data, it will be mostly a re-flight of the original GRACE design, with one important exception: the addition of a laser ranging interferometer (LRI) [7] as a technology demonstrator, which will improve the inter satellite ranging measurement by a factor of 20 . It will be the first time that a laser interferometric ranging measurement will be performed between satellites. As inter-satellite laser ranging is also the basis for the proposed space-based gravitational-wave detector eLISA [8], the development and testing of inter-satellite laser ranging techniques is important not only for the future of space-based Earth gravity measurements, but also for space-based gravitational-wave detection. Indeed, the LRI of GRACE Follow-on has inherited several concepts from eLISA; in turn, the technology developed for the LRI could prove useful for the technical mission design of eLISA, such as allowing the incoming and outgoing beams to be separated without resorting to polarization states, and without changing the phase centre of the measurement. This paper describes the triple mirror assembly (TMA), a central component of the LRI.

\section{The laser ranging interferometer for GRACE follow-on}

The GRACE measurement system consists of a $K$-band ranging system located on the lineof-sight between the centres of mass (CoM) of the two spacecraft. Accelerometers are placed at the CoM of the two spacecraft. Signals from the accelerometers [9] are used to subtract non-gravitational accelerations from the ranging measurement time series. As the LRI is a technology demonstrator, it must not interfere with the existing $K$-band ranging system; nonetheless to minimize the coupling of spacecraft rotational motion into the measurement, the LRI must also measure along the effective line-of-sight between the two spacecraft CoM. The 'racetrack' measurement scheme, depicted in figure 1 and described by Sheard [7], accomplishes this without disturbing the existing $K$-band measurement system. 


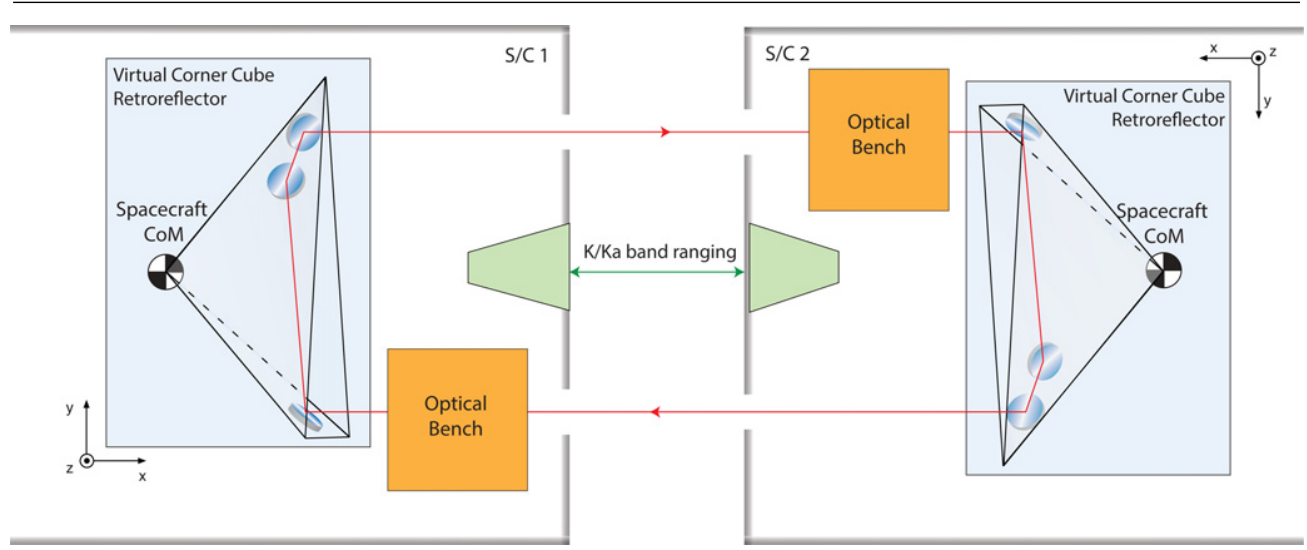

Figure 1. The racetrack measurement principle for the GRACE Follow-on laser ranging interferometer. Spacecraft 1 is on the left, spacecraft 2 on the right. The separation between the spacecraft is approximately $200 \mathrm{~km}$. The optical bench on each spacecraft contains a laser source and optics for measuring the relative phase of the incoming light and the local laser.

In this measurement setup, spacecraft 1 ( $\mathrm{SC} 1$ ) carries a frequency stabilized laser [10], which is routed through a beam router to $\mathrm{SC} 2$. On $\mathrm{SC} 2$, the beam coming from the $\mathrm{SC} 1$ is interfered with a portion of the locally generated laser beam, and the laser on SC2 is offset phase-locked to the incoming beam. This phase-locked light is then sent through the beam router towards SC1. On each spacecraft, a phasemeter (similar to the LISA phasemeter [11]) is used to measure the relative phase of the local and returning beam. The phase measurements from each spacecraft are combined in post-processing to measure the round-trip path length.

For the racetrack measurement scheme to perform at the desired sensitivity of $80 \mathrm{~nm} / \sqrt{\mathrm{Hz}}$ (from 10 to $100 \mathrm{mHz}$, rolling up as $f^{-2}$ below $10 \mathrm{mHz}$ ), the beam router must not contribute more than $20 \mu \mathrm{rad}$ of co-alignment error between the incoming and outgoing beams, in addition to other requirements listed in table 1 and discussed in section 3.1. The TMA design, described in the next section, meets all of these requirements.

\section{The triple mirror assembly}

The simplest method to guarantee that the incoming and outgoing beams are co-aligned is with an optical retro-reflector (a corner cube). For the LRI of GRACE Follow-on, a corner cube whose vertex is located at the SC CoM simultaneously satisfies two critical requirements: the beam co-alignment is always guaranteed by the optical properties of the corner cube; and the total path length (the racetrack measurement) becomes largely insensitive to spacecraft rotations. As an accelerometer is located at the SC CoM (as on the original GRACE satellites), the corner cube vertex cannot physically be co-located there. However, a virtual corner cube can be used. This is achieved by removing those portions of the corner cube not illuminated by the beam. The remaining surfaces, illuminated by the beam and held in place by a suitable support structure, comprise the TMA. This is a form of a lateral transfer retro-reflector [12].

\subsection{Requirements}

The TMA requirements are set by the LRI mission objectives and relevant launch and spacecraft environments. The mission related requirements are listed in table 1. The co-alignment 
Table 1. Requirements on the triple mirror assembly.

\begin{tabular}{ll}
\hline & Requirement \\
\hline Co-alignment (at centre temperature) & $10 \mu \mathrm{rad}$ \\
Co-alignment (thermal stability) & $1 \mu \mathrm{rad} \mathrm{K}$ \\
Vertex position (x, y, z) & $0.5 \mathrm{~mm}$ \\
Vertex position knowledge (x, y, z) & $100 \mu \mathrm{m}$ \\
Free aperture & $20.4 \mathrm{~mm}$ \\
Flatness \& surface quality & $\lambda / 20,10 / 5$ \\
Mass & $\leqslant 1 \mathrm{~kg}$ \\
Atomic oxygen & To withstand flux of $3.2 \times 10^{17} /\left(\mathrm{m}^{2} \mathrm{~s}\right)$ \\
Path length stability & $\leqslant 21 \mathrm{~nm} / \sqrt{\mathrm{Hz}}$ \\
Resonant frequency & $\geqslant 140 \mathrm{~Hz}$ \\
Field of view & $\pm 2 \mathrm{mrad}$ \\
Polarization cross-talk & $<20 \mathrm{~dB}$ \\
Beam separation & $60 \mathrm{~cm}$ \\
\hline
\end{tabular}

requirements are to ensure that adequate laser power is delivered to the distant spacecraft, and requirements on vertex positioning are determined by the coupling of spacecraft rotation into the displacement measurement. In addition to these requirements, the TMA must survive the launch and pre-launch, and so it must be mechanically strong and dynamically rigid enough to withstand a design load acceleration of $100 \mathrm{~g}$ and survive temperatures from -20 to $+45{ }^{\circ} \mathrm{C}$ without suffering permanent damage. The predicted error in co-alignment from the $1 \mathrm{~g}$ release (the difference in gravitationally induced stresses on earth and in orbit) must be less than $3 \mu \mathrm{rad}$ (this means the assembly must be aligned in a g-compensated manner) and the co-alignment requirement must be met in a fully demoisturized state (cf section 4.5.2). The thermal performance requirements are to ensure that the TMA will function adequately over the entire range of the allowable flight temperature, $+10-+30^{\circ} \mathrm{C}$.

In addition to these requirements, the TMA must geometrically fit within the available space in the spacecraft, in such a manner that the vertex can be positioned at the SC CoM. There is an available route that circumnavigates a spherical cold-gas storage tank and avoids other SC components, with a $60 \mathrm{~cm}$ beam separation in $y$, plus a $48 \mathrm{~mm}$ separation in $z$. The TMA has thus been designed for this separation between the incoming and outgoing beams. This path minimizes disruptions to the existing SC design and, critically, does not affect the $K$-band ranging instrument.

\subsection{TMA design}

The prototype TMA design presented here (see figure 2, a colour-coded render) is composed of the three mirrored faces of a corner cube with excess material removed (figure 2(a)), two mounting prisms (figure 2(b)), a carbon-fibre reinforced polymer (CFRP) cylindrical spacer, and two glass inserts (figure $2(c)$ ) bonded into the CFRP cylinder. The mechanical combination of the CFRP spacer and the two inserts comprise the TMA frame (figure $2(d)$ ), and the rest is the mirror assembly. The end faces of the frame are polished flat and parallel after being bonded into the CFRP spacer. Completing the polishing of the frame ends after bonding allows the correction of any distortion of the end faces caused by the epoxy cure process. This permits the mirror assemblies at each end of the frame to be locally referenced to the flat face of the insert during most of the assembly process, rather than requiring $\mu \mathrm{rad}$ metrology over the full beam separation of $60 \mathrm{~cm}$ for the entire assembly process. 


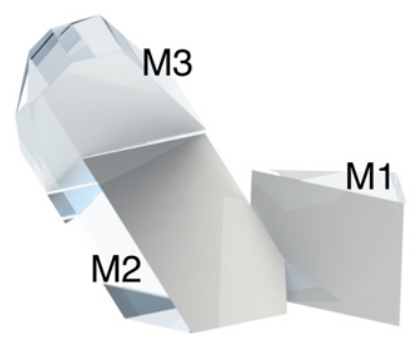

(a)

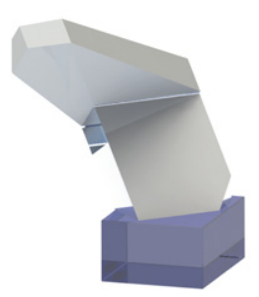

(b)

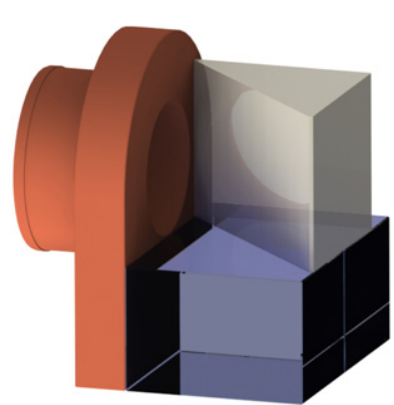

(c)

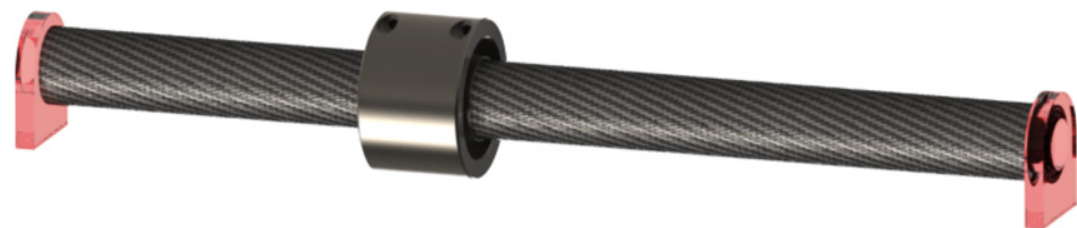

$(d)$

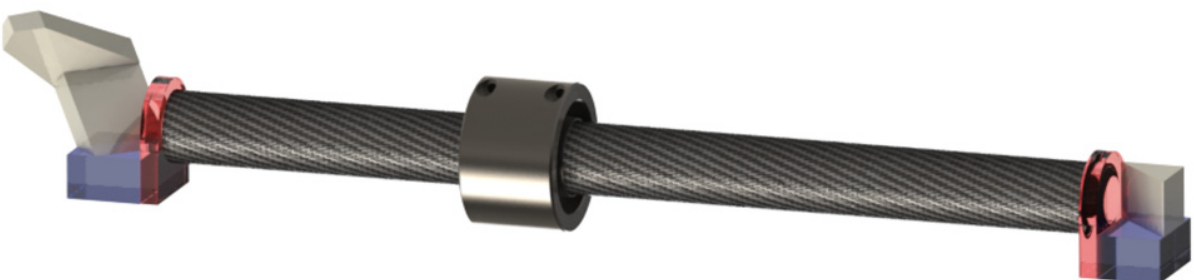

(e)

Figure 2. The parts of the triple mirror assembly. (a) The polished mirrors comprising the corner-cube, here shown together and labelled in order of beam incidence. (b) Mirrors (grey) separated and attached to prisms (blue). (c) Mirror M1 (grey), prism (blue), and glass insert (red). (d) The TMA frame, with end glass inserts shown in red. (e) The triple mirror assembly.

3.2.1. Mirror alignment. The co-alignment of the incoming and outgoing laser beams depends critically on the orthogonality of the reflecting surfaces of the three mirrors; this is the condition for a corner-cube retroreflector. The design mirror normal vectors relative to the spacecraft axes (cf figure 1) are

$$
n_{1}=\left(\begin{array}{c}
1 / \sqrt{2} \\
1 / \sqrt{2} \\
0
\end{array}\right) \quad n_{2}=\left(\begin{array}{c}
0.5 \\
-0.5 \\
-1 / \sqrt{2}
\end{array}\right) \quad n_{3}=\left(\begin{array}{c}
0.5 \\
-0.5 \\
1 / \sqrt{2}
\end{array}\right)
$$

Standard polishing techniques can be used to construct a corner cube to high-precision using surfaces polished to $90^{\circ}$; three regular rectangular prisms can be assembled into a corner cube. This method immediately constrains five of the nine angular degrees of freedom of the three mirrors. The sixth degree of freedom (rotation of one mirror about its normal) does not affect the optical properties of the cube, and the remaining $3^{\circ}$ of freedom are the orientation of the corner cube. 


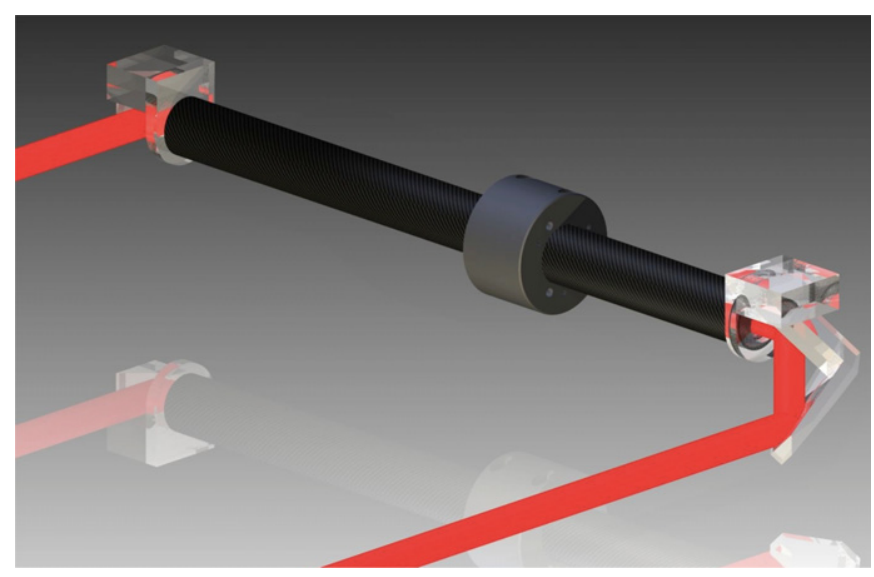

Figure 3. A rendering of the CFRP TMA. This rendering shows the input and output beam paths in red. Also shown here is the retro-fitted titanium bracket.

Constructing a virtual corner cube when the mirrors are spatially separated requires a careful procedure to guarantee the optical performance. In the case of the TMA prototype described here, this performance is achieved by combining a number of optical components which have accurately polished $90^{\circ}$ angles and parallel surfaces, measured using interferometry [13]. Mirror M1 (figure 2(a)) is half of a cube; the active surface of the mirror is at $90^{\circ}$ to the top and bottom (normal to spacecraft $z$-axis), and at $45^{\circ}$ to the sides (spacecraft $x$ and $y$ axes). The active surface of mirror M2 (figure 2(a)) is at $45^{\circ}$ to the 'bottom' surface (normal to spacecraft $z$-axis). The active surface of M2 is also polished to be at $90^{\circ}$ to the surface which will contact the plane of M3; this guarantees that M2 and M3 are orthogonal. In addition to these angles, we exploit the fact that the prisms (figure 2(b)) are polished rectangular to high-precision, and that the end faces of the frame (figure 2(d)) are parallel. A global reference (cf section 4.4) must be used to ensure that the two prisms are aligned in rotation about the spacecraft $y$-axis and height in the spacecraft $z$-axis while being fixed to the frame. The final degree of freedom to ensure coplanarity of the prism 'top' surfaces is constrained by the insert parallelism. This constrains 4 of the 5 required degrees of freedom for the corner cube. The remaining degree of freedom is the relative angular orientation in rotation about the spacecraft $z$-axis of the M1 mirror and the M2/M3 assembly. This final degree of freedom must be measured live relative to the global reference ( $\mathrm{cf}$ section 4.4) as the final step in the assembly.

Finally, the requirement on the position of the vertex of the virtual corner cube sets two more constraints: the separation between the M1 and M2/M3 mirrors must be set to sub-mm precision (which constrains the position in the spacecraft $x$-axis), and the common angular orientation of M1 and M2/M3 (which constrains the vertex position in the spacecraft $y$-axis) must be controlled. The latter is accomplished by ensuring that the non-mirrored faces of M1 are parallel to the faces of the prism supporting M1. Correctly constraining the vertex position in the spacecraft $z$-axis requires correctly orienting the prisms about the spacecraft $y$-axis, during assembly, to some fixed reference on the mounting bracket which serves as the interface to the spacecraft bus. This is discussed further in section 4.6.

The fully assembled TMA is shown (rendered) in figure 3 with ingoing and outgoing beams pictured in red. 


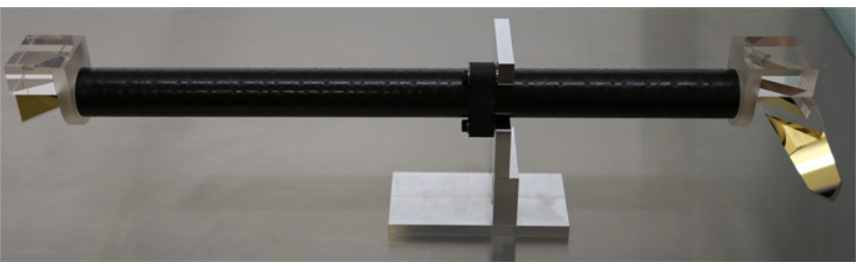

Figure 4. The triple mirror assembly prototype, as built. The mounting bracket shown here is a CFRP bracket made from the same material with the same layup as the tube; this bracket does not adequately distribute the stress on the tube and so the titanium retrofit bracket (shown in 2(e)) was designed and affixed. The aluminium fixture is a stand for laboratory use.

\section{Triple mirror assembly technologies}

\subsection{ULE Mirrors}

Corning ultra-low expansion (ULE) glass is used for the optics and the inserts. The mirrors for the prototype assembly are formed using an unprotected gold coating $(R=0.97 @ 1064 \mathrm{~nm})$. The unprotected gold will not withstand the atomic oxygen in the low-earth orbit space environment, so a protected gold coating will be used for the flight models. Alternatively, multi-layered dielectric coatings are under development, including compensation of surface deformation due to coating stresses.

\subsection{Mechanical frame: CFRP spacer and glass inserts}

The spacer is a CFRP hollow cylinder of length $512 \mathrm{~mm}$, outer diameter of $39 \mathrm{~mm}$, and inner diameter of $35 \mathrm{~mm}$, from Bestcomposites. The weave is UHM640 with LS20 resin, in a $0^{\circ}, 60^{\circ}$, $-60^{\circ}$, quasi-isotropic layup. The coefficients of thermal expansion in the fibre and orthogonal directions are -1.1 and $30 \mathrm{ppm} \mathrm{K}^{-1}$, respectively. The inserts are made from Corning ULE glass.

The frame also includes a bracket for mounting to the SC bus. In figure 4 the bracket shown is made of the same CFRP material as the spacer; during prototyping it was discovered that this design would likely fail during launch, so a titanium bracket was retro-fitted for vibration testing (described in a separate paper [14]); the titanium bracket has also undergone a further redesign, not described in this paper. This redesign also addresses issues relating to spacecraft integration (cf section 4.6).

\subsection{Bonding}

The TMA requires two types of bonds, one at the CFRP-glass interfaces and another at the glass-glass interfaces. For the CFRP-glass interfaces we have used Hysol 9396 epoxy, chosen for its high shear strength. For the glass-glass interfaces, we use hydroxy-catalysis bonding [15-18], chosen for its combination of small bond thickness (thus preserving the alignment properties of the corner cube mirrors, which relies on the polishing), mechanical strength and stability, low stress and hence low distortion, and working time.

\subsection{Reference bar}

To measure the co-alignment at the $\mu \mathrm{rad}$ level, a reference which is aligned to better than this level over $60 \mathrm{~cm}$ is required. An optical reference bar [19], a rectangular $(67 \mathrm{~cm} \times 9 \mathrm{~cm} \times 9 \mathrm{~cm})$ 


\section{Type: Displacement}
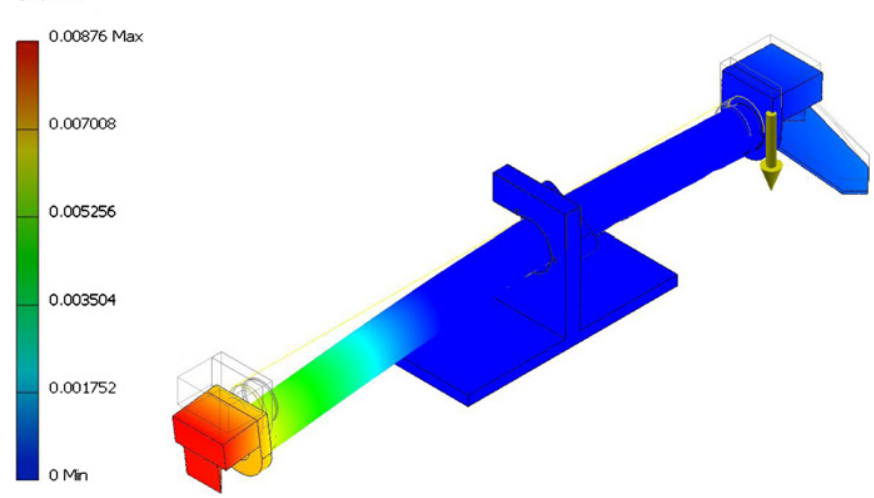

Figure 5. A finite element analysis of the static deformation of the triple mirror assembly when supported near its centre of mass under and experiencing an acceleration of $1 \mathrm{~g}$. This level of deformation leads to a misalignment that exceeds the requirements on the TMA, thus necessitating that the TMA be supported in gravity-compensating mounts when measuring the co-alignment in the laboratory.

fused silica bar polished to better than $500 \mathrm{~nm}$ peak-to-valley over $65 \mathrm{~cm}$, was constructed by Australia's Commonwealth Scientific and Industrial Research Organisation (CSIRO) for this purpose, which leads to a maximum error contribution of approximately $3 \mu \mathrm{rad}$.

\subsection{Assembly}

The assembly must not only guarantee the performance of the TMA on Earth, but must be carried out in such a way that the performance will be maintained in the low-earth orbit environment. This means the assembly procedure must be designed to pre-compensate for the difference in gravity ( $1 \mathrm{~g}$ release) and the difference in humidity between the two environments.

4.5.1. $1 \mathrm{~g}$ release. Designing the assembly procedure to properly account for a $1 \mathrm{~g}$ release is critical, as the TMA must maintain co-alignment on orbit when stresses due to the Earth's gravity have been relieved. For a perfectly assembled TMA on earth, held by its bracket near the TMA CoM with Earth's $g$ pointing along the spacecraft $z$-axis, the total bend in the tube will be approximately $30 \mu \mathrm{rad}$. This exceeds the total co-alignment requirement for the TMA and so must be compensated. Figure 5 shows a finite element analysis of the displacement of the TMA ends when the TMA is supported near the tube centre.

During the assembly, this bending affects the parallelism of the end inserts of the frame, and thus also the prisms. To properly compensate this, metrology to determine the parallelism of the end faces and of the prisms is done with the TMA tube held vertically. Any additional moment about the spacecraft $x$-axis due to the asymmetry of the end inserts themselves, with the TMA held in this position, is negligible.

For the final metrology after assembly (see Fleddermann [14]), the TMA must be supported horizontally, as it is otherwise not possible to measure the parallelism of the incoming and outgoing beams (as they are separated by $60 \mathrm{~cm}$ ). As the masses at the mirror assemblies are a significant component of the total mass, the bending of the tube due to gravity cannot be fully compensated by simply supporting the tube at the Airy points. FE analysis indicated that proper g-compensation required supporting the tube near the end points, along with adding an 
additional 30 grams of mass near the CoM (accomplished using dowel pins in the mounting holes of the bracket).

4.5.2. Moisture release. The CFRP tube can undergo a twisting deformation as it absorbs moisture from the air. The spacecraft environment is very low-humidity, and so for optimal performance the assembly procedure must thus take place in a similarly dry state. Fortunately, the remoisturization time for the CFRP tube is typically several days. It is thus possible to design an assembly procedure where the critical stages take place immediately after demoisturizing through baking. In this manner the TMA will be properly co-aligned when in the low-humidity space environment.

\subsection{Spacecraft integration}

For the triple mirror assembly to fulfil its designed purpose on orbit, it is necessary for the vertex of the TMA to coincide with the CoM of the spacecraft, where an accelerometer is located to measure non-gravitational forces acting on the spacecraft. Accurately placing the vertex at the CoM is required to benefit from the rotation-insensitivity of the TMA about its vertex, which permits a rejection of spacecraft rotation that would otherwise influence the ranging measurement.

This requires constructing the TMA with the vertex at a specified location, referenced to some coordinate axes, to better than $0.5 \mathrm{~mm}$ (cf table 1); furthermore, the position of the TMA vertex must be known to better than $100 \mu \mathrm{m}$, referenced to the same coordinate system. The requirements are determined using estimates of the spacecraft rotational noise and the estimated knowledge of that rotational noise (which comes primarily from onboard star-tracker cameras). Finally, this coordinate system must be easily identifiable by specified markers physically present on the TMA, so that it may be referenced to the spacecraft coordinate system during the integration of the TMA into the spacecraft. The location of the TMA vertex can be determined with a coordinate measurement machine to better than $65 \mu \mathrm{m}$ [20], and the point of minimal rotation to path length coupling (which can differ from the vertex position if the TMA is not perfect, although they will coincide to much better than $100 \mu \mathrm{m}$ if the co-alignment requirement is met) can be determined to better than $20 \mu \mathrm{m}$ by actively rotating the TMA on a hexapod and finding the centre of rotation which minimizes the path length coupling [20].

The design presented here is limited in its integrability into a spacecraft. In particular, the original CFRP bracket shown in figure 4 presented three issues: (1) the possibility of mechanical failure; (2) rotations of the TMA about the spacecraft $y$-axis are not well constrained, and this can lead to a large vertex placement error in the spacecraft $z$-axis; (3) it required mounting at the edge of the spacecraft accelerometer platform, which might lead to stability problems. These issues have all been resolved with a new, updated bracket design.

\section{Measured optical performance}

The coalignment of the TMA prototype described here has been measured [21] using a WYKO 6000 homodyne phase-shifting interferometer, along with a flat reference bar [19] that was used to extend the $150 \mathrm{~mm}$ interferometer aperture to the required $600 \mathrm{~mm}$ of the TMA. For a gravity-compensated two-point mounting close to the TMA endpoints with a centre load of 30 grams, the optimal centre load as noted in section 4.5.1, a root-mean-square coalignment error of $9 \mu \mathrm{rad}$ was measured after subtracting known systematics. This meets the co-alignment 
requirement of less than $10 \mu \mathrm{rad}$ of error at room temperature. For details of the measurement, along with the results of other tests, see Fleddermann et al [14] and Schütze et al [21].

\section{Conclusion}

We have described the detailed design and assembly techniques for a precision optical beam routing assembly for the laser ranging interferometer of the GRACE Follow-on mission. This instrument will provide the first inter-satellite ranging measurement at nm-level precision, and will be the first use of inter-satellite laser interferometry. The technologies developed for this instrument, including the triple mirror assembly described in this paper, represent an important step forward in the use of precision laser optics in space. This is thus an important milestone not only for future GRACE-like missions, but for future space technologies such as formation flying of satellites and inter-satellite laser communications, and future space-based gravitational observatories.

\section{Acknowledgments}

The TMA development was funded by the Australian Space Research Program, an initiative of the Australian Government, with contributions from the German Federal Ministry of Education and Research, the German Aerospace Centre, Australia's Commonwealth Scientific and Industrial Research Organisation, and the Deutsche Forschungsgemeinschaft (DFG) within the Cluster of Excellence QUEST (Centre for Quantum Engineering and Space-Time Research). The authors thank Bill Folkner for invaluable help in the early stages of the TMA design.

\section{References}

[1] Dunn C et al 2003 Instrument of GRACE: GPS augments gravity measurements GPS World 14 16-28

[2] Tapley B D, Bettadpur S, Watkins M and Reigber C 2004 The gravity recovery and climate experiment: mission overview and early results Geophys. Res. Lett. 31 L09607

[3] Schmidt R, Petrovic S, Güntner A, Barthelmes F, Wünsch J and Kusche J 2008 Periodic components of water storage changes from GRACE and global hydrology models J. Geophys. Res.: Solid Earth 113 B08419

[4] Wouters B, Chambers D and Schrama E J O 2008 GRACE observes small-scale mass loss in Greenland Geophys. Res. Lett. 35 L20501

[5] Tiwari V M, Wahr J and Swenson S 2009 Dwindling groundwater resources in northern India, from satellite gravity observations Geophys. Res. Lett. 36 L18401

[6] Watkins M, Flechtner F, Morton P and Webb F 2013 Status of the GRACE follow-on mission Geophys. Res. Abstr. 15 EGU2013-6024

[7] Sheard B S, Heinzel G, Danzmann K, Shaddock D A, Klipstein W M and Folkner W M 2012 Intersatellite laser ranging instrument for the GRACE follow-on mission J. Geod. 86 1083-95

[8] www.elisascience.org/

[9] Touboul P, Willemenot E, Foulon B and Josselin V Accelerometers for CHAMP, GRACE and GOCE space missions: synergy and evolution Bollettino di Geofisica Teorica ed Applicata 401999

[10] Folkner W M et al 2011 Laser frequency stabilization for GRACE-II Proc. of the 2011 Earth Science Technology Forum

[11] Shaddock D, Ware B, Halverson P G, Spero R E and Klipstein B 2006 Overview of the LISA Phasemeter Laser Interferometer Space Antenna: 6th International LISA Symposium (American Institute of Physics Conference Series vol 873) ed S M Merkovitz and J C Livas pp 654-60

[12] Lipkins M 1976 Lateral transfer retroreflectors US Patent 4065204 
[13] Oreb Bozenko, Burke Jan, Netterfield J, Seckold J, Leistner A, Gross M and Dligatch S 2006 Development of precision double corner cubes for the Space Interferometer Mission Proc. SPIE 6292629202

[14] Fleddermann R et al 2014 Testing the GRACE-FO triple mirror assembly Class. Quantum Grav. submitted

[15] Gwo D-H 1998 Ultraprecision bonding for cryogenic fused-silica optics Proc. SPIE 3435 136-42

[16] Elliffe E J, Bogenstahl J, Deshpande A, Hough J, Killow C, Reid S, Robertson D, Rowan S, Ward H and Cagnoli G 2005 Hydroxide-catalysis bonding for stable optical systems for space Class. Quantum Grav. 22 S257-67

[17] Green K, Burke J and Oreb B 2011 Chemical bonding of ultra low expansion glass substrates with aqueous $\mathrm{NaOH}$ solution Mater. Chem. Phys. 130 577-82

[18] Green K, Burke J and Oreb B 2011 Chemical bonding for precision optical assemblies Opt. Eng., Bellingham $\mathbf{5 0} 023401$

[19] Farrant D, Brown J, Seckold J and Dligatch S 2014 A 65-cm silica optical flat reference bar in preparation

[20] Schütze D, Müller V, Stede G, Sheard B S, Heinzel G, Danzmann K, Sutton A and Shaddock D A 2014 Retroreflector for GRACE Follow-On: vertex versus point of minimal coupling Opt. Express at press

[21] Schütze D, Farrant D, Shaddock D A, Sheard B S, Heinzel G and Danzmann K Measuring coalignment of retroreflectors with large lateral incoming-outgoing beam offset Rev. Sci. Instrum. 852014 